\title{
Micro-photoluminescence spectroscopy on heavily-doped layers of silicon solar cells
}

\author{
Hieu T. Nguyen ${ }^{\star}, 1$, Di Yan ${ }^{1}$, Fan Wang ${ }^{2}$, Peiting Zheng ${ }^{1}$, Young Han ${ }^{1}$, and Daniel Macdonald ${ }^{1}$ \\ ${ }^{1}$ Research School of Engineering, College of Engineering and Computer Science, The Australian National University, Canberra, ACT \\ 2601, Australia \\ 2 Department of Electronic Materials Engineering, Research School of Physics and Engineering, The Australian National University, \\ Canberra, ACT 2601, Australia
}

Received ZZZ, revised ZZZ, accepted ZZZ

Published online ZZZ (Dates will be provided by the publisher.)

Keywords Band-gap narrowing, crystalline silicon, heavily-doped silicon, photoluminescence, solar cells.

We report and explain the photoluminescence spectra emitted from silicon solar cells with heavily-doped layers at the surface. A micro-photoluminescence spectroscopy system is employed to investigate the total spectrum emitted from both the heavily-doped layer and the silicon substrate with micron-scale spatial resolution. The two regions of the device give rise to separate photoluminescence peaks, due to band-gap narrowing effects in the highly-doped layer.
Two key parameters - the absorption depth of the excitation wavelength, and the sample temperature - are shown to be critical to reveal the separate signatures from the two regions. Finally, this technique is applied to locally diffused and laser-doped regions on silicon solar cell pre-cursors, demonstrating the potential value of this micron-scale technique in studying and optimizing locally doped regions.
1 Introduction Spectral photoluminescence (PL) has been demonstrated to be a promising and powerful characterization technique in crystalline silicon. By capturing the band-to-band (BB) photoluminescence signal, fundamental parameters of silicon such as the band-to-band absorption coefficient [1-3], radiative recombination coefficient [2,4,5], and temperature [6] and doping dependencies $[7,8]$ of the silicon band gap have been determined. In addition, spectral PL methods have also been employed as a characterization tool in photovoltaics, for example to extract the diffusion length of minority carriers in silicon wafers $[9,10]$ and bricks [11], to quantify the light trapping capability of plasmonic structures [12], to examine the impacts of surface reflectance $[13,14]$ and different carrier profiles [14] on PL spectra, or to evaluate the laser-doped layers of silicon solar cells by applying the band-gap-narrowing effect in heavilydoped silicon $[15,16]$.

Heavily-doped layers, typically formed by dopant diffusion or laser doping, are critical components of a silicon solar cell, as they form the required p-n junction, and allow electrical contact to be made to the device. However, these heavily doped layers are also often a source of significant carrier recombination in solar cells. Thus there is usually a requirement to minimize the surface area of these layers in order to achieve high efficiency solar cells [17]. Therefore, an insightful understanding of the PL spectra emitted from the heavily-doped layers of silicon solar cells with high spatial resolution will facilitate the development of precise characterization tools for silicon photovoltaics.

Micro-photoluminescence spectroscopy has been applied previously on carefully cross-sectioned and polished solar cell pre-cursors $[15,16]$ to reveal the depth profile of highly-doped regions, based on the shift of the PL peak caused by band-gap narrowing. This method is restricted to relatively thick doped layers (tens of microns), such as aluminum-alloyed regions. In this study, we demonstrate a method based on PL spectra measured from above the sample surface, which contain luminescence emitted from both the diffused layer and the underlying silicon substrate. We show that these two regions give rise to distinct PL peaks, due to band-gap narrowing effects in the heavily-doped layer. The method can be applied to detect heavily-diffused regions with thicknesses below $1 \mu \mathrm{m}$, which is more typical for phosphorus and boron-diffused layers in high efficiency

* Corresponding author: hieu.nguyen@anu.edu.au 
silicon solar cells. We then examine two critical parameters allowing an unambiguous identification of the individual components of the combined spectrum emitted by the two layers - the sample temperature and the excitation wavelength. Finally, we demonstrate this method of detecting heavily doped layers on a passivated-emitter rear locallydiffused (PERL) solar cell pre-cursor in which the locally diffused region is a few tens of microns wide. We also apply the method to laser-doped wafers, revealing interesting effects at the edges of the laser doped regions with lateral spatial resolution in the micron range.

2 Background In heavily doped silicon, the intrinsic band gap is reduced [18]. Thus, the PL spectrum emitted from heavily doped silicon is shifted to longer wavelengths, i.e. lower energies. Wagner $[7,8]$ utilized this phenomenon to determine the extent of band-gap narrowing in silicon, using epitaxially grown silicon layers whose doping densities were homogeneous depthwise.

For solar cells, heavily-doped sub-surface layers are typically formed by thermal diffusion or laser doping, which give rise to relatively shallow (typically less than $1 \mu \mathrm{m}$ ), and inhomogeneously doped layers depthwise. In these cases, if an appropriate excitation wavelength is employed, this excitation light will be absorbed in both the diffused layer and the underlying silicon substrate. These two layers will then both emit PL spectra. The total detected PL spectrum is a superposition of the spectra from the two layers, with the spectrum from the heavily-doped layer being energy-shifted by varying degrees via band-gap narrowing.

However, at room temperature, the BB PL spectrum from silicon is relatively broad [2,3]. Thus, when the PL spectra from the two regions are combined, the contributions of the individual components from each layer are difficult to distinguish. On the other hand, at cryogenic temperatures, the BB peaks are much sharper due to reduced lattice vibrations [2,3]. Therefore, the BB peaks from the two layers may be resolved clearly from each other, allowing us to unambiguously detect the presence of the diffused layers.

2 Experimental details The investigated samples were phosphorus-doped n-type float zone silicon wafers whose surfaces were mechanically polished and chemically etched to remove saw damage. Their resistivities were between 1 and $10 \Omega . \mathrm{cm}$, corresponding to background doping levels between $6 \times 10^{15}$ and $6 \times 10^{14} \mathrm{~cm}^{-3}$. These samples were heavily doped with boron from a $\mathrm{BBr}_{3}$ vapor source (for forming the $\mathrm{p}^{+}$layer) or phosphorous from a $\mathrm{POCl}_{3}$ vapor source (for the $\mathrm{n}+$ layer) in a quartz tube furnace at high temperatures between $900-1000{ }^{\circ} \mathrm{C}$ with various drive-in times to achieve different doping profiles in the diffused layers. The samples were then immersed in HF solution to remove the borosilicate or phosphosilicate glass layers. The doping profiles of the $\mathrm{p}^{+}$and $\mathrm{n}+$ layers were measured using the electrochemical capacitance-voltage (ECV) technique, and are plotted in Fig. 1b for four representative $\mathrm{p}^{+}$boron diffused samples. The sample size is about $1 \times 1 \mathrm{~cm}^{2}$, with a thickness of about $400 \mu \mathrm{m}$.

The diffused solar cell pre-cursor and laser-doped sample were phosphorus-doped n-type float zone silicon wafers with background doping levels of $2 \times 10^{15}$ and $7 \times 10^{14} \mathrm{~cm}^{-3}$, respectively. The diffused pre-cursor went through the standard fabrication steps for PERL n-type cells, which achieve efficiencies above $20 \%$, as described in more detail elsewhere [19]. The rear locally phosphorus-diffused regions were $75 \mu \mathrm{m}$ in diameter on $300 \mu \mathrm{m}$ spacing. The dopant source used for the laser-doped sample was a commercial boron spin-on-dopant solution. The laser doping was achieved using a HeCd laser (325-nm wavelength, 4.05$\mathrm{J} . \mathrm{cm}^{-2}$ fluence, 25-ns pulse duration, and $320-\mu \mathrm{m}$ square beam diameter).

The micro-PL spectroscopy system employed in this study is a Horiba T64000 equipped with a confocal microscope. The incident laser beam was focused into a spot of about $1 \mu \mathrm{m}$ in diameter on the samples using a $50 \times$ objective reflective lens. The emitted PL signal was collected by a liquid-nitrogen-cooled InGaAs array detector. The spectral response of the entire system was calibrated with black body thermal radiation. The employed lasers were a continuous wave diode-pumped solid-state (DPSS) laser and a diode laser. The DPSS laser wavelength was $532 \mathrm{~nm}$, corresponding to an absorption depth of about $3.3 \mu \mathrm{m}$ at $79 \mathrm{~K}$ (calculated from Ref. 20), and on-sample average intensity was $6 \mathrm{~mW}$. The diode laser wavelength was $830 \mathrm{~nm}$, corresponding to an absorption depth of about $45 \mu \mathrm{m}$ at $79 \mathrm{~K}$, and its power was adjusted to be the same as that of the DPSS laser. The sample temperature was controlled with a liquid-nitrogen cryostat. More details on the setup can be found in Ref. 21. We simulated the heating effect of the excitation laser on the samples using the LCPSim software [22,23], and found that the local temperature at the center of the illuminated spot was increased less than $6^{\circ}$. In addition, we varied the excitation power from 0.7 to $8.5 \mathrm{~mW}$ and captured the spectra at $300 \mathrm{~K}$; and then varied the temperature from 300 to $305 \mathrm{~K}$ and captured the spectra with $8.5-\mathrm{mW}$ excitation power. We found that the change in the BB spectrum shape of the former case was very much less than that of the latter, and thus conclude that the degree of local heating due to the excitation laser is insignificant. We note that the samples had no surface passivation layers present during the measurements.

3 Results and Analysis First, we examine the combined spectra emitted from the diffused samples at $79 \mathrm{~K}$. Fig. 1a shows the normalized PL spectra from four $\mathrm{p}^{+}$diffused samples, corresponding to two categories: heavy and deep profiles (samples 1 and 2), and light and shallow profiles (samples 3 and 4). The spectrum from an undiffused sample is also given for comparison. The dopant profiles of the diffused layers are plotted in Fig. 1b. For the case of heavy and deep profiles (samples 1 and 2) in Fig. 1a, two distinct 
peaks at around $1130 \mathrm{~nm}$ and $1160 \mathrm{~nm}$ correspond to the BB peak of the silicon substrate (Si BB) and the BB peak of the diffused layer (diffused BB), respectively. The wavelength of the diffused BB peak moves towards longer wavelengths when the doping concentration at the surface is higher (sample 1). In addition, we can observe the transverse-optical phonon replica (shifted by $\sim 60 \mathrm{meV}$ ) of the diffused BB peak (diffused BBTO) at around $1225 \mathrm{~nm}$. This phonon replica is the analogue of the transverse-optical phonon replica of the Si BB peak (Si BBTO) at around $1195 \mathrm{~nm}$, which is also depicted in Fig. 1a for the case of light and shallow profiles (samples 3 and 4), and for the undiffused case. For the lighter diffusions, the signal of the diffused BB peak is not strong enough to mask the phonon replica of the Si BB peak.

Note that the energy difference between the two BB peaks is around $\sim 30 \mathrm{meV}$, which is smaller than the expected energy shift of $\sim 60 \mathrm{meV}$ for the doping concentrations $\sim 1 \times 10^{19} \mathrm{~cm}^{-3}$ [24] in comparison to intrinsic silicon. The reason is that the silicon substrate itself is under high injection, thus its BB peak is also partly affected by bandgap narrowing. The average injection level in the silicon substrate was estimated to be between $1 \times 10^{17}$ and $1 \times 10^{18}$ $\mathrm{cm}^{-3}$, for which the silicon band gap is reduced by $\sim 30-40$ $\mathrm{meV}[25,26]$. We note that this band-gap narrowing effect should not be confused with the donor-acceptor pair (DAP) luminescence in compensated silicon [27], since the DAP peak is detected at lower temperatures, and is shifted towards higher energies as the net doping concentration increases [28], whereas the diffused BB peak here is shifted towards lower energies (longer wavelengths). Fig. 1c plots the wavelength of the diffused BB peak versus the peak doping concentration in the diffused layer. Since the diffused BB peak is broad, the peak wavelength was determined by taking the average of all points having PL intensities larger than $99 \%$ of the maximum value. The peak wavelength clearly increases as the peak doping concentration increases, supporting the suggestion that the peak is due to the bandgap narrowing effect.

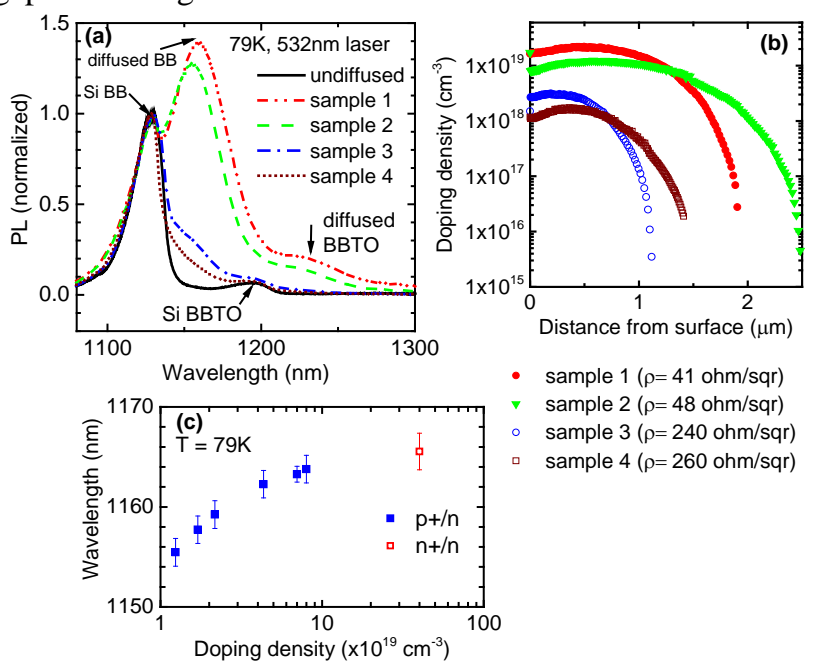

Figure 1 (a) Normalized PL spectra of the four samples with different boron diffusion profiles, measured with the 532-nm laser at
$79 \mathrm{~K}$. The spectrum of an undiffused sample is also given for comparison. (b) The corresponding diffusion profiles with the sheet resistances indicated in brackets. (c) Wavelength of the diffused BB peak, along with one standard deviation error bars, versus peak doping density in $\mathrm{n}+$ and $\mathrm{p}+$ layers on $\mathrm{n}$-type substrates.

Next, we demonstrate the importance of temperature and excitation wavelength on detecting the diffused layers on silicon substrates. Fig. 2a shows the normalized spectra of sample 1 at different temperatures. As the temperature increases, the two BB peaks are thermally broadened, and eventually combine together into a very broad peak having a maximum in between the two low temperature BB peaks. Also, the insert compares the spectra of sample 1 and the undiffused sample at $300 \mathrm{~K}$, which have maxima at 1146 and $1135 \mathrm{~nm}$, respectively. This wavelength shifting is due to the aggregation of the diffused and Si BB peaks in sample 1. These two spectra are indistinguishable on the lower wavelength side. However, on the higher wavelength side, the heavily doped region gives rise to a clear shoulder, although not a distinct peak. The spectra from the light and shallow profiles (samples 3 and 4) are closer to that of the undiffused sample, and thus the presence of their diffused layer is much more difficult to observe at room temperature. Note that this signature is opposite to the effects caused by non-uniform excess carrier profile and surface reflectivity, in which the higher wavelength side of the normalized spectrum is not affected due to the very long absorption depth of photons in this wavelength region, whereas the low wavelength side is affected more significantly at higher temperatures [14].

In addition, Fig. 2b shows the spectra of sample 1 excited with both 532-nm and 830-nm lasers. Since the absorption depth of the $830-\mathrm{nm}$ laser is about $45 \mu \mathrm{m}$ at $79 \mathrm{~K}$ [20], the laser light is mostly absorbed in the silicon substrate, and thus the signature of the diffused layer is suppressed significantly. This suggests that only the diffused BB peak may be observed if the sample is illuminated with an ultra-violet laser, for example with a 325-nm HeCd laser having an absorption depth of less than $0.01 \mu \mathrm{m}$ at $79 \mathrm{~K}$. The results from this figure confirm that the peak at around $1160 \mathrm{~nm}$ is emitted from the layer close to the surface, and is associated with the diffused layer in this study. Therefore, employing an appropriate excitation wavelength and performing the measurements at low temperatures are essential to observe the two separate BB peaks.

We also captured the spectra at numerous different locations for several samples, and found that despite a small change in the relative PL intensity, the spectrum shape from each sample is consistently unchanged regardless of the locations, indicating a high degree of spatial homogeneity of the diffused layers. This property could be potentially applied to directly characterize the spatial homogeneity of the diffused layers on silicon solar cells and cell pre-cursors, which could be particularly useful for studying locally-diffused or laser-doped regions. 


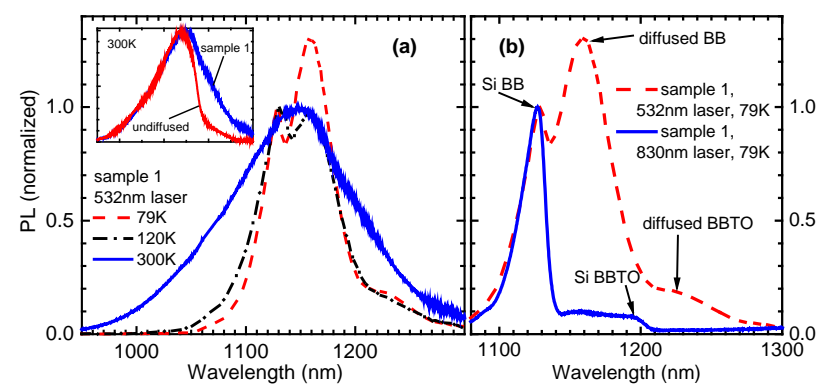

Figure 2 Normalized PL spectra of sample 1 (a) at different temperatures excited with 532-nm laser, and (b) excited with 532-nm and $830-\mathrm{nm}$ lasers at $79 \mathrm{~K}$. The insert compares the normalized spectra of sample 1 and the undiffused sample at $300 \mathrm{~K}$.

Furthermore, we examine the effect of excitation power on the spectrum shape. Unlike the undiffused silicon sample whose spectrum shape does not change with a small variation of excitation power, the spectrum shape of the diffused sample displays a notable dependence on excitation power. Fig. 3a shows the normalized spectra of sample 1 with different excitation powers from the 532-nm laser at $79 \mathrm{~K}$. The normalized Si BB peak is saturated whereas the normalized diffused BB peak is reduced with increasing excitation power. The reason is that the average injection level was estimated to be between $1 \times 10^{17}$ and $1 \times 10^{18} \mathrm{~cm}^{-3}$, whereas the doping density in the diffused layer near the surface is around $2 \times 10^{19} \mathrm{~cm}^{-3}$, while that in the silicon substrate is around $1 \times 10^{15} \mathrm{~cm}^{-3}$. Thus, the silicon substrate is under high injection level whereas the diffused layer is still in the low injection regime. Therefore, the dependence of the BB peak intensities on the excitation power is different for the two layers. Since the PL intensity $\sim\left(\mathrm{N}_{\mathrm{D}}+\Delta \mathrm{n}\right) \times \Delta \mathrm{n}$ where $\mathrm{N}_{\mathrm{D}}$ is the doping density and $\Delta \mathrm{n}$ is the excess carrier density [14], the PL signal is expected to be approximately linearly proportional to $\Delta \mathrm{n}$ in the diffused layer, but should be a quadratic function of $\Delta \mathrm{n}$ in the silicon substrate. When the excitation power is increased, the PL signal of the diffused layer increases more slowly than that of the substrate, and thus the normalized diffused BB peak is less pronounced. When the dopant concentration of the diffused region is reduced, this effect is reduced.

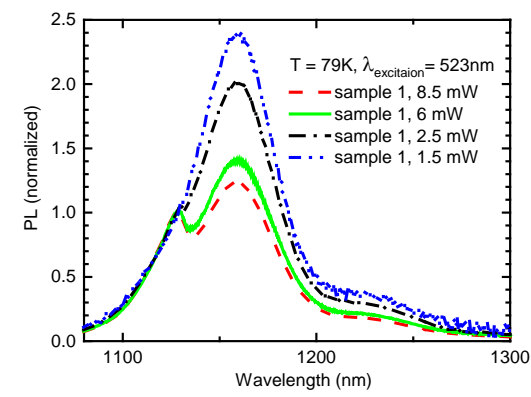

Figure 3 Excitation power dependence of normalized PL spectra of sample 1, measured with the 532-nm laser at $79 \mathrm{~K}$.

Finally, we demonstrate this method on a locally $n+$ diffused region of a PERL n-type silicon solar cell pre-cursor, and on an edge of the $\mathrm{p}^{+}$region of a laser-doped sample. The profile of the localized $n+$ region on the rear is given in Fig. 4a. This profile was measured using the ECV technique on a control wafer undergoing the same diffusion process. The 3D optical surface image of the edge of the laser-doped $\mathrm{p}^{+}$region is given in Fig. $4 \mathrm{~d}$. The raised edge region is about $5 \mu \mathrm{m}$ wide and $2 \mu \mathrm{m}$ higher than the surface of the $\mathrm{p}^{+}$and undoped regions. Figs. 4b and 4c show the normalized spectra captured from the $\mathrm{n}+$ and undiffused regions of the precursor, and from the three regions ( $\mathrm{p}^{+}$, undoped, and edge) of the laser-doped sample. For the diffused pre-cursor, in Figs. 4a and 4b, the diffused profile (heavy but shallow) lies between the two categories examined above, so the diffused $\mathrm{BB}$ peak in this case is somewhere between the two cases in Fig. 1a. For the laser-doped sample, in Fig. 4c, the diffused $\mathrm{BB}$ peak of the edge is notably higher than that of the $\mathrm{p}^{+}$ region, and its peak energy is shifted slightly to longer wavelengths compared to that of the $\mathrm{p}^{+}$region. These features indicate that the edge is doped more heavily than the $\mathrm{p}+$ region. In our experimental setup, this technique is effective for diffused layers having doping concentrations as low as $1 \times 10^{18} \mathrm{~cm}^{-3}$ (sample 4 in Fig. 1a). In practice, the doping levels of the $\mathrm{n}+$ and $\mathrm{p}+$ regions in solar cell applications are generally higher than this concentration. Therefore, this technique can provide a measure of the lateral extent of heavily-doped layers with micron-scale resolution, and may be extended to allow quantitative estimates of the dopant concentrations in such heavily-doped regions.
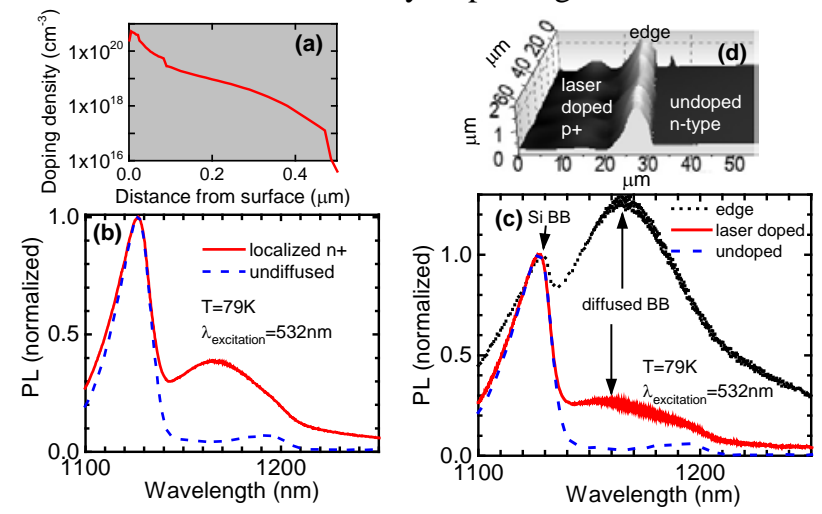

Figure 4 Normalized PL spectra (b) of the localized + and undiffused regions of the PERL solar cell pre-cursor and (c) of the $\mathrm{p}^{+}$, undoped, and edge regions of the laser-doped sample, excited with the 532-nm laser at $79 \mathrm{~K}$. (a) Diffusion profile of the localized n+ region of the pre-cursor. (d) 3D optical surface image of the edge of the laser-doped $\mathrm{p}+$ region.

4 Conclusions Utilizing the doping dependence of the band gap in silicon, we have demonstrated a contactless and non-destructive spectral photoluminescence technique to detect thin heavily-doped layers on silicon substrates with micron-scale spatial resolution. In addition, we have empirically shown the importance of appropriate excitation wave- 
lengths and sample temperature in order to separate the signatures of the two layers. Finally, we have demonstrated this technique on a silicon solar cell pre-cursor whose diffused regions are several tens of microns wide, and on a laserdoped damaged edge whose width is only several microns.

Acknowledgements This work has been supported by the Australian Research Council (ARC) and the Australian Renewable Energy Agency (ARENA) through research grant RND009. The Australian National Fabrication Facility is acknowledged for providing access to some of the facilities used in this work. The authors are in debt to Prof. H. Tan for providing access to the spectroscopic equipment and for commenting on the manuscript, Dr. A. Fell for assisting with the LCPSim simulation, and Dr. S. P. Phang and Dr. F. E. Rougieux for assisting with some measurements.

\section{References}

[1] E. Daub and P. Würfel, Phys. Rev. Lett. 74, 1020 (1995).

[2] T. Trupke, M. A. Green, P. Würfel, P. P. Altermatt, A. Wang, J. Zhao, and R. Corkish, J. Appl. Phys. 94, 4930 (2003).

[3] H. T. Nguyen, F. E. Rougieux, B. Mitchell, and D. Macdonald, J. Appl. Phys. 115, 043710 (2014).

[4] P. P. Altermatt, F. Geelhaar, T. Trupke, X. Dai, A. Neisser, and E. Daub, Appl. Phys. Lett. 88, 261901 (2006).

[5] H. T. Nguyen, S. C. Baker-Finch, and D. Macdonald, Appl. Phys. Lett. 104, 112105 (2014).

[6] W. Bludau, A. Onton, and W. Heinke, J. Appl. Phys. 45, 1846 (1974).

[7] J. Wagner, Phys. Rev. B 29, 2002 (1984).

[8] J. Wagner, Phys. Rev. B 32, 1323 (1985).

[9] P. Würfel, T. Trupke, T. Puzzer, E. Schäffer, W. Warta, and S. W. Glunz, J. Appl. Phys. 101, 123110 (2007).

[10] J. A. Giesecke, M. Kasemann, M. C. Schubert, P. Würfel, and W. Warta, Progress in Photovoltaics: Res. \& Appl. 18, 10 (2011).

[11] B. Mitchell, M. K. Juhl, M. A. Green, and T. Trupke, IEEE Journal of Photovoltaics 3, 962 (2013).

[12] C. Barugkin, Y. Wan, D. Macdonald, and K. R. Catchpole, IEEE Journal of Photovoltaics 3, 1292 (2013).

[13] C. Schinke, D. Hinken, J. Schmidt, K. Bothe, and R. Brendel, IEEE Journal of Photovoltaics 3, 1038 (2013).

[14] H. T. Nguyen, F. E. Rougieux, S. C. Baker-Finch, and D. Macdonald, IEEE Journal of Photovoltaics 5, 77 (2015).

[15] R. Woehl, P. Gundel, J. Krause, K. Rühle, F. D. Heinz, M. Rauer, C. Schmiga, M. C. Schubert, W. Warta, and D. Biro, IEEE Trans. Elec. Devices 58, 441 (2011).

[16] P. Gundel, D. Suwito, U. Jäger, F. D. Heinz, W. Warta, and M. C. Schubert, IEEE Trans. Elec. Devices 58, 2874 (2011).

[17] A. Wang, J. Zhao, and M.A. Green, Appl. Phys. Lett. 57, 602 (1990).

[18] R. A. Abram, G. J Rees, and B. L. H. Wilson, Adv. Phys. 27, 799 (1978).

[19] Y. Wan, C. Samundsett, T. Kho, J. McKeon, L. Black, D. Macdonald, A. Cuevas, J. Sheng, Y. Sheng, S. Yuan, C. Zhang, Z Feng, and P. J. Verlinden, in Proc. IEEE 40th Photovoltaic Spec. Conf., Denver, Colorado (2014).

[20] M. A. Green, Sol. Energy Mater. Sol. Cells 92, 1305 (2008).

[21] H. T. Nguyen, F. E. Rougieux, F. Wang, H. Tan, and D. Macdonald, "Micron-scale deep-level spectral photoluminescence from dislocations in multicrystalline silicon,” IEEE Journal of Photovoltaics, accepted for publication (2015).

[22] A. Fell and G. P. Willeke, Appl. Phys. A 98, 435 (2010).
[23] A. Fell, "Modelling and simulation of laser chemical processing (LCP) for the manufacturing of silicon solar cells", Ph.D dissertation, Fraunhofer Institute for Solar Energy Systems (2010).

[24] J. Wagner and J. A. del Alamo, J. Appl. Phys. 63, 425 (1988).

[25] J. W. Slotboom and H. C. de Graaff, Solid-State Electronics 19, 857 (1976).

[26] M. Y. Ghannam and R. P. Mertens, Microelectronic Engineering 19, 691 (1992).

[27] M. Tajima, T. Iwai, H. Toyota, S. Binetti, and D. Macdonald, J. Appl. Phys. 110, 043506 (2011).

[28] B. D. Rezgui, J. Veirman, S. Dubois, and O. Palais, Phys. Status Solidi A 209, 1917 (2012)). 